\title{
A Prospective Study of the Neonatal Outcome in Diabetic Pregnancies
}

\author{
Lakshmi M, Anil H and Basavarajaiah DM* \\ Department of Paediatrics, Bangalore Medical College and Research Institute, \\ Bengaluru, India \\ *Corresponding Author: Basavarajaiah DM, Department of Paediatrics, Bangalore \\ Medical College and Research Institute, Bengaluru, India.
}

DOI: $10.31080 /$ ASPE.2020.03.0223
Received: January 28, 2020;

Published: February 11, 2020

(C) All rights are reserved by Basavarajaiah

DM., et al.

\section{Abstract}

India, being the second leading nation of diabetic subjects (69.2 million), has become the "Diabetic Capital of the World" harboring around 4 million women with GDM alone. IDF, 2013 has been estimated that, the total of 21.4 million live births will be affected with hyperglycemia in pregnancy. Though, the number of live births were affected by hyperglycemia in pregnancy, the rate has declined slightly from 21.4 million to 20.9 million in 2015, the adverse perinatal outcomes in (85.1\%) cases are still due to GDM, (7.4\%) due to other types of diabetes first diagnosed in pregnancy, and the remaining (7.5\%) cases due to diabetes detected prior to pregnancy. Many challenges were pose to extrapolate the incidence of GDM at population level in global platform as well as national level and also which is cited by the less literature in Indian context, since more research should be inculcate to formulate the decision about the exposed population. In this intervene, the present study aims to know the occurrence of metabolic and haematological complications in infants of diabetic mothers and also correlate the occurrence of congenital anomalies in infants of diabetic mothers. It is a hospital based prospective cross sectional observational study, a total 100 neonates born to diabetic mothers were evaluated. The maternal history and details of glycemic control was recorded. Neonatal history, physical examination for the presence of major congenital anomalies and birth injuries was performed. Laboratory investigations like blood glucose, calcium, complete hemogram and echocardiography was done routinely in all babies. The results were compared and evaluated in both pregestational and gestational diabetes, the effect of glycemic control on the various neonatal complications were analyzed. The median age of diabetic mothers was 27 with SD 2.1 years. Gestational diabetes was seen in (74\%) while pre gestational diabetes (26\%). Hypoglycemia was the commonest metabolic complication documented (63\%) cases, occurred within 6 hours of birth and polycythemia was the commonest hematological abnormality was seen in (45\%) cases. Hypoglycemia was strongly associated with congenitalanomalies and macrosomia. Congenital anomalies were observed in (33\%) cases where cardiac malformations were common of which ASD and PDA predominated. Birth injuries were seen in (11\%) cases in which Erb's palsy was seen in 6 cases and almost all injuries were due to macrosomia, which was seen in (34\%) of all neonates born to diabetic mothers. There was a strong association of high levels of HbAIC levels with hypoglycemia, macrosomia and congenital malformations. Mortality in our study was $3 \%$ and all cases were died due to congenital anomaly. Women with pre gestational diabetes have an increased risk of congenital anomalies and mortality. This study also showed a high percentage of neonatal complications due to poor glycemic control in pregnancy. Hypoglycaemia in IDMs was strongly associated with congenital anomalies and macrosomia which was the major cause for morbidity and mortality in IDMs.

Keywords: Gestational Diabetes; Pregestational; HbA1C; Macrosomia; Hypoglycemia; Congenital Anomalies

\section{Introduction}

The diabetes is a chronic metabolic disorder due to either insulin deficiency (relative or absolute) or due to peripheral resistance to the action of insulin [1]. In broad sense gestational diabetes is defined as it is carbohydrate intolerance of variable severity with onset or first recognition during pregnancy $[2-5,15]$. The World Health Organization (WHO) estimates that, the prevalence of diabetes mellitus (DM) will show a alarming stage to drew up 150 to 333 million by 2025 [6-10]. This alarming calendar year will increase in the prevalence of DM, it will occur mainly in the develop- ing country like India and south Asian countries and the child bearing age would be most affected parameter [2]. Since, the prevalence of GDM in India significantly varies from 3.8 to $21 \%$ in different parts of the country, depending on the geographical locations and diagnostic methods used [3]. The recent data on the prevalence of GDM in our country prevalence was $16.55 \%$ by WHO criteria of $2 \mathrm{hr} P \mathrm{PG} \geq 140 \mathrm{mg} / \mathrm{dl}$ [4]. Neonatal mortality rate in diabetic pregnancies is five times more than that of non-diabetic pregnancies [10]. Neonates born to diabetic mothers are at higher risk for developing congenital anomalies, small for gestational age (SGA), 
macrosomia, metabolic abnormalities like hypoglycaemia, hypocalcaemia, hypomagnesemia, haematological complications like hyperbilirubinemia, hyper viscosity secondary to polycythemia, respiratory distress due to antagonistic effect of hyperinsulinemia on cortisol mediated surfactant synthesis [5]. The prevalence of congenital anomalies in infants of diabetic mothers is $6-10 \%$ [12]. Most common fetal structural defects were associated with maternal diabetes are cardiac malformations followed by neural tube defects, renal agenesis and skeletal malformations [13]. Cardiac anomalies specific to GDM include asymmetrical septal hypertrophy [15]. Cardiomegaly occurs in 30\% and cardiac failure in 5-10\% of neonates [14]. Macrosomia results showed increased incidence of operative deliveries and shoulder dystocia with potential brachial plexus injury was seen [5]. Strict glycemic control during peri conception and during labour reduces the incidence of anomalies and neonatal hypoglycaemia respectively [8]. Since, the incidence of gestational diabetes is increasing which in turn increasing the incidence of adverse neonatal outcome, there is a need for study of diabetes cases are up to 422 million worldwide. India ranks among top 3 countries with diabetic population. While the numbers gone up to 11.9 million in 1980 to 64.5 million in 2014 in India [16]. Prevalence of diabetes has two fold for men in India $3.7 \%$ to $9.1 \%$ ) and by $80 \%$ among women in India ( $4.6 \%$ to $8.3 \%$ ) [11]. According CDC report, 2014 prevalence of gestational diabetes is more as increase up 9.2\% [12]. There is a higher level of prevalence of gestational diabetes at 22 to $25 \%$ as allow to debate the issues at worldwide prevalence number 15\%. (IDF,2014) As per the International Diabetes Federation (IDF), Diabetes Atlas 2015, a total 1 in 7 births are deleteriously affected by GDM [18]. India, being the second leading dweller of diabetic subjects (69.2 million), has become the "Diabetic Capital of the World" harboring around 4 million women with GDM alone [21]. IDF, 2013 has estimated a total of 21.4 million live births to be affected with hyperglycemia in pregnancy [20]. Though the number of live births affected by hyperglycemia in pregnancy has declined slightly from 21.4 million to 20.9 million in 2015 , the adverse perinatal outcomes in $85.1 \%$ of cases are still due to GDM, 7.4\% due to other types of diabetes first diagnosed in pregnancy, and the remaining $7.5 \%$ of cases due to diabetes detected prior to pregnancy [13]. Many challenges were listed to extrapolate the incidence of GDM at population level at global as well as national level and also cited by the less literature in Indian context, since more research should be support for formulate the decision about the exposed population. In this intervene, the present study aims to know the occurrence of metabolic and haematological complications in infants of diabetic mothers and also correlate the occurrence of congenital anomalies in infants of diabetic mothers.

\section{Methodology}

A cross sectional observational study conducted at Department of OBG, BMCRI, Bengalore. A total 100 patients selected based on the following inclusion criteria; Singleton neonates of diabetic mothers exclusion criteria; Neonates of diabetic mothers with medical complications such as heart disease, and renal disease. Neonates of diabetic mothers with pregnancy induced hypertension and eclampsia and twin neonates of diabetic mothers. With prior informed consent was obtained from the parents/guardians of neonates under study. The maternal data was recorded including age, parity, gestational age, mode of delivery and the outcome. Maternal diabetes mellitus diagnosis was done based on diabetes in pregnancy study group in India (DIPSI) criteria; it requires a 2 hours venous plasma glucose $\geq 140 \mathrm{mg} / \mathrm{dl}$ in the non fasting Oral Glucose Tolerance Test (OGTT). Their glycemic control in the form of HbA1C done at the first visit recorded. The neonatal data including 'APGAR' score, birth weight, sex and gestational age, weight for gestation was extracted, a screening the cases on the basis of physical examination in the presence of major congenital anomalies. Serial blood glucose levels were taken at 1, 2, 3, 6, 12, 24 and 48 hours by using glucometer. All neonates, a serum calcium and complete haemogram were done routinely at 24 hours of age and later if required. Echocardiography was done (all the infants). The salient criteria was adopted for the selection of sample viz hypoglycaemia is defined as blood glucose of less than $40 \mathrm{mg} / \mathrm{dl}$, hypocalcaemia as serum calcium level of less than $7 \mathrm{mg} / \mathrm{dl}$. Polycythemia- hematocrit higher than $65 \%$ or haemoglobin ( $\mathrm{Hb}$ ) concentration more than 20 $\mathrm{gm} / \mathrm{dl}$. Macrosomia- birth weight more than 90th percentile (large for gestational age, LGA), fetal growth restriction as birth weight less than the 10th percentile (small for gestational age, SGA). Major congenital anomaly - any of the followings: lethal, life-shortening life-threatening, requires major surgery or affecting in a significant way the quality of life. Collected data was analyzed by using Minitab and epi- info statistical soft ware. Data was obtained from patients with greater accuracy, lesser standard error. Logistic regression analysis was used to test the hypothesis.

\section{Results}

The results revealed that, the majority of cases (74\%) of infants were born to mothers with gestational diabetes mellitus (GDM) is found to be statistically significant with mean age of the mother (27.65 with SD 2.55 years) and (64\%) mothers were conceived at the age of 26-30 years. Among the 100 neonates we tested our objective of interest, the neonates (31.08\%) who born GDM mother, the birth weight weighs from 3 to $3.49 \mathrm{kgs}$ (61.53\%). In DM cases we sequentially birth weight was recorded and it was found to be $>4 \mathrm{Kg}(3.6 \%)(\mathrm{p}<0.05)$. A total $(11 \%)$ neonates were recorded at low birth weight $(<2.5 \mathrm{~kg})$ the results showed that, there is no significant differences between the age and GDM. An approximately (61\%) neonates were delivered by caesarean section $\mathrm{p}<0.05$; (89\%) neonates were born term and majority (60\%) neonates were born with appropriate gestational age of the mother. A total (67.31\%) GDM; AGA (57.69\%) out of 26 of overt DM are LGA.

The resulted findings were apparently correlate with all haematological parameter $(\mathrm{p}<0.05)$. Since, the hypoglycaemia $(63 \%)$ 
p $<0.05$ drew up in GDM cases and most common metabolic abnormality was seen in neonates who born to diabetic mother, the occurrence of the event of metabolic abnormality (MA) showed statistically differ among infants who born to overt DM mothers ( $p$ $<0.05)$. Of which 63 IDMs with hypoglycaemia $(82.5 \%)$ occurred within 6 hrs of life, (49.2\%) in the first hour 11(17.46\%) cases were seen between 6 and 24 hrs respectively. The hypocalcaemia was seen in $36(48.63 \%)$ cases $(\mathrm{p}<0.01)$, inclusion with $6(23.07 \%)$ cases were deliriously expressed hypocalcaemia $(\mathrm{p}<0.01)$. This study showed that, the most common haematological abnormality significantly correlated irrespective of the age of the mother and the onset of mode of delivery. The polycythemias (45\%), the occurrence showed significantly differ among infants born to GDM. Hyperbilirubinemia estimated values were increased significantly more in infants of GDM (44.73\%). Of which 40 infants $12(30.00 \%)$ cases were developed the complications on day 3 of life, 6 (15\%) cases occurrence exhibits between 4 th and 5 th postnatal day. The most common congenital anomaly in neonates was recorded, the cardiovascular abnormalities $30(91 \%)$ and $19(73.07 \%)$ cases were found in overt DM mother. The HbA1C levels were $>6.5 \%$ estimated in GDM p $<0.01$. The common cardiovascular abnormality was seen in (16) cases ostium secundum ASD, PDA followed by VSD and only one case had asymmetrical septal hypertrophy $\mathrm{p}>0.01$. When compared to infants of $11(14.86 \%)$ cases of GDM mothers $p$ $<0.05$ it was highly associated with post complications. CNS anomaly seen in $2(7.6 \%)$ cases and renal anomaly only $1(3.8 \%)$ case was recorded during the study period. This study showed that, the majority of neonates born to diabetic mothers were significantly associated with Erb's palsy (6\%). The total mortality (3\%) was seen in both population (GDM and overt), a total $2(7.7 \%)$ cases found overt GDM mortality.

\section{Discussion}

In India, while the most pressing and commonest problem is high risk pregnancies, of which diabetes is a common medical complications of pregnancy. Women with this complication can be divided into those who were known to have diabetes before pregnancy (pregestational) and those diagnosed during pregnancy (gestational). WHO estimates an alarming increase in the prevalence of DM, which will occurs mainly in the developing regions of the world and the child bearing age would be most affected [25]. The presence of diabetes before pregnancy is a risk factor bears on the adverse neonatal outcomes, especially congenital anomalies. In Indian scenario, there is an increased risk of immediate complications in IDMs including Congenital anomalies, macrosomia, hypoglycemia. The long term complications include an increased rate of adolescent obesity, impaired glucose tolerance or diabetes mellitus. Delivery of an infant with a major malformation has become the leading cause of perinatal mortality in pregnancies complicated by diabetes and these complications are the consequences of poorly controlled diabetes both preconceptionally as well as early in pregnancy. Prior studies on the prevalence of infant malformations associated with different types of maternal diabetes during pregnancy are limited [9]. And most of the studies have been limited to analysis of women with either gestational or pregestational diabetes but not both types were compared. The published literature showed regarding outcome of diabetic pregnancies varies from one region to other sites. The present study correlates with other studies in which the occurrence of infants born to gestational diabetic mothers is (74\%) and that of pregestational diabetic mothers is (26\%). Of this 26 all were of type-II diabetes. Haider Shirazi., et al. opined that, an increase in occurrence of pre gestational diabetes is because of recent increase in the prevalence of DM especially affecting the child bearing age. Present study, a majority of the IDMs were males (74\%) and significantly associated with age of the mother. The Cardiac anomalies were seen in (90.9\%) of the cases which was also similar study done by Joanne Yang., et al. In our study, the neonates with congenital heart disease, most common was ASD, PDA followed by VSD and one case had asymmetrical septal hypertrophy. There was a significant association between hypoglycemia in IDMs and congenital anomalies. Another significant association was observed between congenital anomalies and $\mathrm{HbA} 1 \mathrm{C}$ levels $>6.5 \%$. The occurrence of congenital anomalies is higher in the present study (similar to Haider shirazi., et al. [6]) when compared to other Studies mentioned above. This difference correlate may be due to routine screening of all the IDMs by echocardiography. The low rate in other studies could have been due to the fact that women in whom glucose intolerance develops after mid pregnancy do not expose the embryo to hyperglycemia during the period of embryogenesis and therefore do not have any increase in congenital malformations. The $\mathrm{HbA} 1 \mathrm{C}$ has been considered to be a good indicator of the glycemic control of the mothers. In our Study (75\%) of the diabetic mother had HbA1C $>6.5 \%$ micro/dL. This level was significant associated with Macrosomia, congenital anomalies. We observed that (73\%) of congenital anomalies and $25 / 34$ of macrosomia was associated with HbA1C levels $>6.5 \%$ micro/dL. Various studies pertaining to the above intervention have also contributed that women with poor glycemic control before and during pregnancy and the fetal hyperinsulinemia, directly contribute to the occurrence of congenital anomalies and macrosomia. In the present study, the presence of mortality was $(3.0 \%)$, the primary cause of death in IDM was congenital anomalies. Two cases were died due to complex (CCHD) and other child was died due to sepsis with meningocoele. The high mortality rate was observed in the study done by Joenne., et al. due to because of septic shock.

\section{Conclusion}

Our results suggests that, the morbidities/complications in IDMs such as macrosomia, birth injuries and congenital anomalies had a significantly associated with hypoglycemia in neonates and poor glycemic control in mothers. It is of concern that, there is not a significant improvement in the neonatal outcome despite improvement of care for diabetic mothers recently. There was a strong association of high levels of HbA1c levels with hypoglycaemia, mac- 
rosomia, LGA and ASH. Cardiac anomalies were predominant and constituted about higher rate of all the congenital anomalies.

\section{Bibliography}

1. Dutta DC. "Diabetes mellitus in pregnancy". In: Dutta DC. The text book of Obstetrics. 7th ed. New Delhi: New central book agency (2006): 281-287.

2. WHO. Diabetes mellitus Fact sheet No. 138. WHO fact sheet No.138 Online (2002).

3. Shah S., et al. "Diagnosis and management of Gestational Diabetes Mellitus". Indian guidelines 3:78-79

4. Havilah Polur K., et al. "Diabetes in Pregnancy Study Group in India (DIPSI)- A Novel Criterion to Diagnose GDM". International Journal of Biochemistry Research and Review 10.1 (2016): 1-6.

5. Carlo WA and Ambalavanan N. "The Endocrine system". In: Kligeman RM, Stanton BF, Geme JW, Schor NF, editors. Nelson textbook of Pediatrics. New Delhi: Elsevier 1 (2015): 897-898.

6. Haider Shirazi., et al. "Neonatal Outcome of Diabetic Pregnancy”. Annals of Pakistan Institute of Medical Sciences 6.2 (2010) 107-112.

7. Joanne Yang., et al. "Fetal and neonatal outcomes of diabetic pregnancies”. The Journal of Obstetrics and Gynecology 108.1 (2006): 644-650.

8. Patt MJ., et al. "Outcome of diabetic pregnancies". Diabetic Medicine 20 (2003): 82-83.

9. Gillan Hawthorne., et al. "Prospective population based survey of outcome of pregnancies in diabetic women: result of Northern diabetic pregnancy Audit 1994". BMJ 2 (1997): 279-281.

10. Farooq MU., et al. Noor Specialist Hospital Makkah, Kingdom of Saudi Arabia Bahawal Victoria Hospital, Bahawalpur- Pakistan". International Journal of Endocrinology Metabolism 3 (2007): 109-115.

11. Jeanne S., et al. "Maternal Diabetes Mellitus and Infant Malformations". The American College of Obstetricians and Gynecologists 100.5 (2002): 655-659.

12. WHO World health day, India among top 3 countries with high diabetic population. Lancet study, annual report.

13. IDF - International Diabetes Federation. Annual Report (2016).

14. Diabetes care. The Journal of Clinical and Applied Research and Education 39.1 (2016): 321-322.
15. Williams obstetrics text book of obstetrics, 24th edition, New Delhi (2014): 2351-1388.

16. Cloherty. Diabetes in pregnancy: In John p cloherty: manual of neonatal care, 7th edition (2016): 11-23.

17. Avery's. Diabetes in pregnancy: Avery's diseases of the newborn, 9th edition (2012): 88-100.

18. Metzger BE., et al. "International Association of Diabetes and Pregnancy Study Groups Recommendations on the Diagnosis and Classification of Hyperglycemia in Pregnancy". Diabetes Care 33 (2010): 676-682.

19. Vandorsten JP., et al. "NIH consensus development conference: diagnosing gestational diabetes mellitus”. NIH State-of-theScience Conference Statement 29 (2013): 1-31.

20. Sharma D., et al. "Asymmetrical septal hypertrophy and hypertrophic cardiomyopathy in infant of diabetic mother A reversible cardiomyopathy". Medical Journal of Dr. D.Y. Patil Vidyapeeth 7.12 (2016): 257-260.

21. Laura J., et al. "Cardiovascular disease risk in the offspring of diabetic women: the impact of the intrauterine environment". Experimental Diabetes Research (2012).

22. N Freinkel. "Of pregnancy and progeny". Diabetes 29.12 (1980): 1023-1035

23. Hod M., et al. "Prevalence of congenital anomalies and neonatal complications in the off springs of diabetic mothers in Israel”. Israel Journal of Medical Science 27.8 (1991): 498-502.

24. Ibrahim A., et al. "Perinatal morbidity and mortality in offspring of diabetic mothers in Qualif, Saudi Arabia". European Journal of Obstetrics and Gynecology and Reproductive Biology 65 (1996): 165-169.

25. Diagnosis and classification of diabetes mellitus. American diabetes association. Diabetic care 29.1 (2006): 256-258.

26. Shefali AK., et al. "Pregnancy outcomes in pre-gestational and gestational diabetic women in comparison to nondiabetic women--A prospective study in Asian Indian mothers (CURES-35)". Journal of the Association of Physicians of India 54 (2006): 613-618.

27. Aslam M., et al. "Clinical spectrum of infants of diabetic mothers in hospitalized deliveries at Lahore". Pakistan Journal of Pathology 12.1 (2001): 5-8.

28. White P. "Classification of obstetric diabetes". American Journal of Obstetrics and Gynecology 130 (1978): 228-230. 
29. Silva Idos S., et al. "Birthweight and other pregnancy outcome in a cohort of women with pre-gestational insulin-treated diabetes mellitus. Scotland". Diabetic Medicine 95.22 (1979): 440-447.

30. Schaefer-Graf UM., et al. "Patterns of congenital anomalies and relationship to initial maternal fasting glucose levels in pregnancies complicated by type 2 and gestational diabetes". American Journal of Obstetrics and Gynecology 182 (2000): 313-320.

\section{Assets from publication with us}

- Prompt Acknowledgement after receiving the article

- Thorough Double blinded peer review

- Rapid Publication

- Issue of Publication Certificate

- High visibility of your Published work

Website: www.actascientific.com/

Submit Article: www.actascientific.com/submission.php Email us: editor@actascientific.com

Contact us: +919182824667 